\title{
FLUCTUATIONS OF REFRACTION IN DEVELOPING CATARACT.
}

\author{
Charles Zimmermann, M D., F. A. C. S.
}

MILWAUKEE, WISCONSIN.

This paper discusses the physiologic and pathologic changes of the crystalline lens in their infuence upon refraction, illustrated by a case of developing cataract observed for many years. It was read before the Wisconsin State Medical Soclety, October 3rd, 1918.

The important part which the crystalline lens normally plays in the refraction of the eye makes it easily conceivable that certain changes of the lens must lead to, alterations of refraction. These changes are physiologic and pathologic. The physiologic changes are a natural accompaniment of the growth (with the advancing years in the course of life) of the individual, and are of great interest for the understanding of the pathology of the lens. Most important are the alterations of the dimensions and shape, consistency, and coloration of the lens. During the whole of life new lens fibers are formed from cells at the meridianal rows of the equator of the lens, so that its volume and weight increase, from the twenty-fifth to the sixty-fifth year by one-third, its diameter by one-tenth. (Priestley Smith. ${ }^{2}$ )

The increase of the volume by this new formation of fibers preponderates over the apparent simultaneous loss of the water content of the lens which produces changes of the nucleus. In consequence of the larger volume of the lens the anterior chamber of older people generally is markedly more shallow. The growth of the lens influences its shape, from the almost spherical form of the lens, if left to itself, of the newborn, to the less curved anterior surface of the senile lens, whose sagittal diameter is smaller than its equatorial diameter. Perhaps the acquired hyperopia at higher age is due to this flattening of the anterior surface of the lens.

Physiologic alterations of consistency are due to sclerosis of the lens, a process which may be compared with the cornification of the epithelium. It is characterized at first by a gradually greater hardness of the more centrally located lens fibers from loss of water.
A cross section of the senile lens fre'quently shows the nucleus dry without luster, the cortex moist and glistening. $\mathrm{Hess}^{2}$ found in some cases the whole nuclear portion converted into a homogeneous mass, in which no single fibers were visible. The sclerosis increases the refractive index. Halben ${ }^{3}$ showed that loss of water alone increases the refractive index of the lens. Whether the higher index in sclerosis is due only to loss of water or also to chemical alterations of the nucleus, e. g., the greater amount of the more refracting cholesterin (Jacobson, Matthiesen), is still uncertain. The nucleus seems to grow with advancing age by supplanting the soft cortex. If this is excessive, it may develop into brunescent cataract, whose brown color differs from the normal senile lens only in degree, not in kind.

Probably in consequence of abnormal loss of water another pathologic condition with apparently normal shape of the lens is created, viz., an abnormally great difference between the central and peripheral parts of the lens which may amount to a difference in refraction of from 10 to 16 diopters. Sometimes the turning of the ophthalmoscopic mirror in such cases shows a migration of the illuminated field in the central parts opposite to that in the peripheral parts. The center of the pupil may appear less bright than the periphery, and the border between them may be indicated by a dark shadow in form of a crescent or a ring. The ophthalmoscopic examination thru the central area of the pupil may reveal more or less high myopia and thru the peripheral area a lower degree of myopia, emmetropia, or hypermetropia. From the similarity of these phenomena with those of true lenticonus, Demicheris ${ }^{4}$ termed them spurious lenticonus. 
They occur in perfectly clear lenses or in lenses that may remain clear for a long time, but also as precursors of, or in combination with, cataract.

Vision may be considerably reduced, so that extraction is indicated, but in some cases it may be improved by correction of the nuclear myopia. Hess showed that from the 25th year with advancing age the difference between the index of the nucleus and that of the adjoining cortex constantly increases. The refraction of rays at the surface of the nucleus, at equal difference of index, must be the greater the greater the curvature of this surface. As the difference of index at higher age, in which this phenomenon exclusively occurs, is considerable, relatively slight differences in the curvature of the nucleus could essentially influence the refraction. Altho these differences of shape of the surface of the nucleus have so far not been exactly investigated, Hess considers their possibility, a priori, as not too remote to serve in the explanation of the phenomenon.

Another disease of the lens without opacity consists in the formation of radial clefts immediately under the capsule, which may remain transparent for a long time. They may be invisible in certain positions of the ophthalmoscope, but on slightly tilting the mirror may shine with a lustre and again appear as darker streaks. Vision may be more or less impaired, and the patient may complain of polyopia and metamorphopsia due to development of lenticular astigmatism. Gradually the contents of these clefts may become opaque and produce cataract. The affection resembles the first stage of naphthalin poisoning of the rabbit, in which Hess showed that the cataract is preceded by the formation of spokes clear as glass. In this stage, volume and weight of the lens are regularly increased. Becker ${ }^{b}$ considered the fine, totally reflecting clefts in the deeper strata of the cortex as the first symptom perceptible during life of incipient senile cataract. Hess, however, proved that these clefts do not lie in the deeper strata of the cortex, but closely under the capsule. He consid- ers this proof important for the understanding of the development of cataract. As is well known, the most frequent form of senile cataract is the subcapsular cortical cataract.

With regard to pathologic alterations of the epithelium Hess examined a large series of eyes affected with various kinds of cataract. The only changes which he could find with certainty in the great majority of these eyes with senile opacities, consisted in more or less numerous irregularly bordcred, vesicular vacuoles, of which he could not say whether they lay between epithelium and capsule, or closely under the epithelium, between this and the cortical fibers. " He never found them in eyes with perfectly transparent lenses, but especially in comparatively early stages of subcapsular cataract, sometimes scarce, sometimes in such quantities that they gave the appearance of fine vesicular foam immediately under the capsule. $\mathrm{He}$ thinks that they cannot be due to anything else but accumulation of small quantities of clear fluid under the capsule, or the epithelium.

Likewise with regard to the occurrence of clear clefts previous to the development of supranuclear cataract, Hess describes a visibly not opaque lens, in which he found a bright reflecting line parallel to the equator between cortex and nucleus, composed of numerous minute clear formations, and also a large number of finest fissures between the apparently normal fibers of the most peripheral layers of the nucleus. It gave the impression as if clear fluid had accumulated in very small quantities in these formations. Hess believes that at the beginning of cataract an intussusception of fluid takes place with the microscopically demonstrated destruction of the epithelial cells, to which he attributes the pathogenesis of subcapsular cataract and the young cortical fibers, which not only anatomically but also in their biologic properties are the more related to the capsular epithelium the younger they are.

To this corresponds the increase of volume of the lens in incipient cataract, 
evidenced by the greater shallowness of the anterior chamber,was also found experimentally in the first stages of naphthalin cataract. Several cases were attributed to this stage of intumescence of the lens in which, in predisposed eyes, glaucoma was brought on by swelling of the lens. The myopia preceding or accompanying the formation of cataract has been ascribed to these changes. By intussusception of water into the cortex its index will be lowered and thereby the total index increased, and also the curvature of the lens may be increased.

In the progress of cataract to maturity the lens gives out fluid to the surroundings. Its volume and weight decrease and with this the depth of the anterior chamber becomes again normal, with decrease of refraction.

As an illustration of the refractive changes due to pathologic alterations of the lens, I should like to report one of a number of cases which I had under observation for many years :

An otherwise healthy, strong man showed, when first examined at the age of 52, emmetropia, and presbyopia. In his 64th year his sight became impaired. He had developed myopia and incipient cataract, at the peripheral cortex of both eyes. Vision was corrected by $\mathrm{R} .-3.50, \mathrm{~L} .-1.00$, for reading $R .-1.50, \mathrm{~L}+0.75$. At $65, \mathrm{~V} . \mathrm{R}$. $20 / 200$, V. L. $20 / 100$. His myopia had increased and was complicated by astigmatism. The ophthalmometer showed R. $-1: 00180^{\circ}$, L. $-0.75180^{\circ}$. V. was corrected with $R .-5.00=-0.50$ cyl. $90^{\circ}$ to $20 / 30$, L. $-1.00=2.00$ cyl. $90^{\circ}$ to $20 / 20$. For reading $R$. -3.50 -0.50 cyl. $90^{\circ}$, L. -1.00 cyl. $90^{\circ}$.

In certain positions of the opthalmoscope the fundus of each eye was clearly visible, in others not, on account of sclerosis of the nucleus of the lens. The peripheral opaque spokes had not much changed. The anterior chamber was shallow. There was monocular polyopia.
In each of the following years the spheres and cylinders had to be increased, vision gradually decreasing even under correction. At the age of 70 he received the maximum, viz.: $\mathrm{R}$. $-10.00=-2.00$ cyl. $90^{\circ}, \mathrm{V} .=20 / 100$, L. $-6.00=-4.50$ cyl. $90^{\circ}, \mathrm{V} .=20 / 50$. At $72 \mathrm{~V}$. with these glasses was the same. V. without glasses was fingers at 6 feet.

The ophthalmoscopic examination revealed $R$. clear red reflex, dimmed in the center by the sclerotic nucleus, details visible, no changes of the retina. Cataract not progressed. Left Eye: The same picture of scletosis with slight opacities at the equator. Sugar was found in the urine for the first time, but there were no other diabetic symptoms. It certainly had nothing to do with the affection of the lens.

At 75, details of fundus could be seen in left eye, not in the right. The sclerosis of the nucleus had increased. The swelling of the lens had decreased as indicated by the less shallow anterior chamber, and also refraction, which was now: R. $-9.00=-1.00$ cyl. $90^{\circ}, V .=20 / 100$, L. $-6.00=$ $-3.75 \mathrm{cyl} 1.90^{\circ}, \mathrm{V} .=20 / 50$, i. e., vision had remained the same. It had not changed in the next four years, but myopia and astigmatism at 79 had further decreased to R. $-7.00, \mathrm{~V} .=20 / 100, \mathrm{~L}$. $-5.00=-2.00$ cyl. $90^{\circ}, \mathrm{V} .=20 / 50$.

At $81 \mathrm{~V}$. with these glasses was, $R$. $20 / 200$, a little less, V. L. 20/70. The ophthalmoscope showed $R$. faint red reflex, some opaque cortex on temporal side, clear on nasal side, nucleus opaque. Left: Cortex pretty clear except a few spokes, nucleus diffusely opaque, but details of fundus faintly visible. With $R,-2.50$ he reads large print; with L. -2.00 cyl. $90^{\circ}$, No. 1 Jaeger at 6 inches, No. 2 at 9 inches, and No. 4 at 11 inches. The patient was a .very assiduous reader, and read with these glasses for many hours every day without discomfort.

\section{REFERENCES.}

1. Priestley Smith. Pathology and Treatment of Glaucoma, 1891, p. 164.

2. Hess, C. Pathologie des Linsen-Systems.

3. Halben. Graefe's Archiv für Ophthalmologie, vol. 57, p. 277.

4. Demicheri. Annales d'Oculistique, vol. 113.

5. Becker. Zur Anatomie der gesunden und kranken Linse, 1883. 